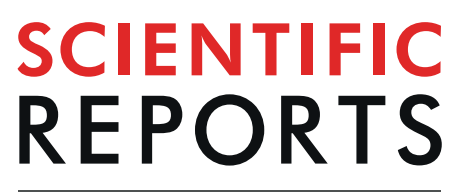

natureresearch

Check for updates

\title{
Soluble programmed death-ligand 1 rather than PD-L1 on tumor cells effectively predicts metastasis and prognosis in soft tissue sarcomas
}

\author{
Kunihiro Asanuma ${ }^{1 凶}$, Tomoki Nakamura ${ }^{1}$, Akinobu Hayashi ${ }^{2}$, Takayuki Okamoto ${ }^{3}$, \\ Takahiro lino ${ }^{1}$, Yumiko Asanuma ${ }^{1}$, Tomohito Hagi ${ }^{1}$, Kouji Kita ${ }^{1}$, Kouichi Nakamura ${ }^{1}$ \& \\ Akihiro Sudo ${ }^{1}$
}

The soluble form of PD-L1 (sPD-L1) is related to a poor prognosis in various cancers. Comparisons of SPD-L1 and PD-L1 expressed on tumor cells in soft tissue tumor patients have not been reported. The purpose of this study was to analyze serum SPD-L1 and PD-L1 levels in soft tissue tumor patients. A total of 135 patients with primary soft tissue tumors were enrolled in this study. The sPD-L1 level was quantitatively measured by enzyme immunoassay, and PD-L1 expression on high grade sarcoma cells was analyzed immunohistologically. There were no significant differences in SPD-L1 levels between benign (48) and soft tissue sarcoma (STS) patients (87). In STS, the high sPD-L1 (>44.26 pg/mL) group had significantly lower metastasis-free survival (MS) and lower overall survival (OS) than the low SPDL1 group ( $\leq 44.26 \mathrm{pg} / \mathrm{mL}$ ) at 5 years using the log-rank test. On multivariate Cox proportional hazard analysis, the high SPD-L1 group had significant differences in MS and OS compared to the low SPD-L1 group. Between positive and negative immunostaining groups, recurrence-free survival (RS), MS, and OS were not significantly different. No correlation was found between immunostaining and sPD-L1 with the Kappa coefficient. The sPD-L1 concentration could predict future metastasis and prognosis in STS patients. High SPD-L1 in STS patients may be a target for treatment with checkpoint inhibitors.

Soft tissue sarcomas (STSs), which are derived from heterogeneous malignant neoplasms arising in the mesenchymal connective tissues, comprise $<1 \%$ of adult malignancies. Although the treatment approach, including surgery, radiotherapy, and combination chemotherapy has improved, more than $40 \%$ of cases have lethal postoperative metastatic recurrence ${ }^{1}$. Recently, attention has been focused on using immunological control points in the cell for immunotherapy in cancer. The immune response is usually in a balance between stimulatory and inhibitory signals. Programmed death-ligand 1 (PD-L1: B7-H1 or CD274), a 40-kDa transmembrane glycoprotein, is known as a primary ligand of PD-1. The interaction of PD-L1 and programmed death 1 (PD-1) can induce T-cell tolerance ${ }^{2}$, T-cell apoptosis ${ }^{3}$, and T-cell exhaustion ${ }^{4}$, leading to evasion of the host immune response and tumor aggravation. Some studies reported that high PD-L1 expression in tumor tissues was related to a poor prognosis in various malignant tumors, including non-small cell lung cancer ${ }^{5}$, ovarian cancer ${ }^{6}$, renal cell carcinoma ${ }^{7}$, melanoma ${ }^{8}$, breast cancer ${ }^{9}$, and STS ${ }^{10}$. Thus, it is recognized that PD-L1 expression affects tumor behavior and prognosis.

In addition, the soluble form of PD-L1 (sPD-L1) in blood has also attracted much attention. The associations of sPD-L1 with the clinical characteristics of various malignant tumors were studied, along with histological PD-L1 expression in tumor tissues. High sPD-L1 is related to a poor prognosis in various cancers, such as renal cell carcinoma ${ }^{11}$, hepatocellular carcinoma ${ }^{12,13}$, esophageal cancer ${ }^{14}$, lung cancer $^{15}$, gastric cancer ${ }^{16-18}$, rectal can$\operatorname{cer}^{19}$, and lymphoma ${ }^{20,21}$. However, no study of sPD-L1 in soft tissue tumor patients and its relationship to prognosis has been reported.

The clinical data showing elevated SPD-L1 and a poor prognosis suggested that aggressive tumors may release and increase sPD-L1 or sPD-L1, making tumor cells aggressive. Given this, we hypothesized that there might be

${ }^{1}$ Department of Orthopedic Surgery, Mie University School of Medicine, Tsu City, Mie, Japan. ${ }^{2}$ Department of Pathology, Mie University School of Medicine, Tsu City, Mie, Japan. ${ }^{3}$ Department of Pharmacology, Faculty of Medicine, Shimane University, Izumo, Shimane, Japan. ${ }^{\bowtie}$ e-mail: kasanum@gmail.com 


\begin{tabular}{|c|c|c|c|c|c|}
\hline Characteristics & & $\begin{array}{l}\text { Healthy } \\
\text { volunteers (10) }\end{array}$ & Benign (48) & STS (87) & p-value \\
\hline \multirow{2}{*}{ Sex } & Male & 2 & 25 & 47 & \multirow{2}{*}{$* 0.126$} \\
\hline & Female & 8 & 23 & 40 & \\
\hline Age & Average (SD) & $51.4(12.5)$ & $54.2(13.7)$ & $63.4(15.1)$ & ${ }^{\#} \mathrm{P}<0.001$ \\
\hline sPD-L1 & Average (SD) & $34.2(10.3)$ & $46.6(24.7)$ & $61.7(58.2)$ & ${ }^{*} 0.017$ \\
\hline $\begin{array}{l}\text { Characteristics in benign } \\
\text { and STS patients }\end{array}$ & & $N(135)$ & sPD-L1 average(SD) & p-value & \\
\hline \multirow{2}{*}{ Sex } & Male & 72 & $55.0(31.7)$ & \multirow{2}{*}{$* * 0.095$} & \\
\hline & Female & 63 & $57.7(64.4)$ & & \\
\hline \multirow{2}{*}{ Age } & $\leq 60 \mathrm{y}$ & 61 & $48.4(28.3)$ & \multirow{2}{*}{$* * 0.228$} & \\
\hline & $>60 y$ & 74 & $62.8(61.1)$ & & \\
\hline \multirow[t]{2}{*}{$\begin{array}{l}\text { History of other malignant } \\
\text { tumors }\end{array}$} & - & 111 & $54.3(36.1)$ & \multicolumn{2}{|l|}{$* * 0.324$} \\
\hline & + & 24 & $65.3(88.4)$ & & \\
\hline
\end{tabular}

Table 1. Characteristics of patients with soft tissue tumors. Sex, age, malignancy, and sPD-L1 values were evaluated by *Fisher's exact test and the \#Kruskal-Wallis test (upper table). sPD-L1 values were compared for each parameter by the **Mann-Whitney test (lower table).

a relationship between the soluble sPD-L1 level and the prognosis of STS patients. The purpose of the present retrospective study was to evaluate correlations between serum sPD-L1 levels and clinicopathological parameters and to elucidate whether SPD-L1 levels and PD-L1 expressed on tumor cells can be used to distinguish the malignant phenotype in soft tissue tumor patients and to predict recurrence, metastasis, or prognosis in STS patients.

\section{Results}

Characteristics of the study population. The clinical and pathological characteristics of the study population are summarized in Table 1. Age and sPD-L1 levels were significantly different between healthy volunteers, the patients with benign tumors and the patients with STS. Although age distribution was different, sPD-L1 levels of STS were significantly high and those of healthy volunteers were low. Box plot of sPD-L1 was shown in Supplementary Fig. S1. The histopathological diagnoses of the 48 benign tumors were 17 lipomas, 15 schwannomas, 5 fibromatoses, 3 myxomas, 3 tenosynovial giant cell tumors, 2 leiomyomas, and 3 others, while those of the 87 STSs were 39 liposarcomas (23 well-differentiated liposarcomas (WLSs), 12 dedifferentiated liposarcomas (DLSs), and 4 myxoid liposarcomas (MLSs)), 14 myxofibrosarcomas (MFSs), 11 undifferentiated pleomorphic sarcomas (UPSs), 9 leiomyosarcomas (LMSs), 5 synovial sarcomas (SSs), 4 malignant peripheral nerve sheath tumors (MPNSTs), and 5 others. All patients with benign tumors underwent tumor resection, and 86 patients with STSs received treatment (wide resection 57 patients, marginal resection 24 patients, intralesional resection 3 patients, ion beam radiotherapy 2 patients) (Table 2). No treatment was performed for 1 patient with an MPNST; this patient was excluded from the prognostic analysis. Although female, patients over 60 years old and those with a history of other malignant tumors had higher sPD-L1 levels, there was no significant difference in sPD-L1 levels for characteristics in benign and STS patients (Table 1).

Characteristics of the STS population. The clinical and pathological characteristics of the STS patients are shown in Table 2. The average SPD-L1 levels in STS patients were higher in females, those over 60 years old, with superficial tumors, with trunk tumors, or with histopathological high-grade tumors, but the differences were not significant. By histopathological subgroups, average (standard deviation) sPD-L1 levels were: MPNST 85.5 (74.2) pg/mL; MFS 86.2 (116.6) pg/mL; UPS 55.8 (30.1) pg/mL; SS 51.6 (14.9) pg/mL; WLS 54.4 (43.8) pg/mL; DLS 55.7 (30.2) pg/mL; MLS 44.8 (22.0) pg/mL; LMS 50.8 (19.0) pg/mL; and others $72.0(64.8) \mathrm{pg} / \mathrm{mL}$ (Supplementary Fig. S2). According to the AJCC classification of STSs, 27 patients were classified as stage I, 15 were classified as stage II, and 44 were classified as stage III. The average SPD-L1concentrations tended to be higher with higher stages than with lower stages, but the difference was not significant.

Recurrence, metastasis, and dead of disease in the STS group. The median follow-up in malignant patients was 42.9 months (range 1.1-417 months). During the period of this study, 18 patients developed recurrence (recurrence group), 28 patients developed metastasis (metastasis group), and 19 patients died of disease (DOD group). The recurrence group showed higher sPD-L1 concentrations than the no recurrence group, but the difference was not significant. The metastasis group and the DOD group had significantly higher sPD-L1 concentrations than the no metastasis group and the no DOD group, respectively (Table 3 ). Additionally, 10 cases were followed-up by measuring SPD-L1 concentrations at the operation for recurrence or metastasis. In 2 cases, sPD-L1 concentrations were decreased, and in 2 other cases, sPD-L1 concentrations were at almost the same levels. In the 6 other cases, sPD-L1 concentrations were increased by recurrence or metastasis (Supplementary Fig. S3).

To confirm the diagnostic accuracy of sPD-L1 for identifying metastasis and DOD, ROC analysis was performed by evaluating the area under the curve (AUC). The AUCs for identifying metastasis and DOD were 0.700 (95\%CI 0.579-0.822) and 0.682 (95\%CI 0.543-0.820), respectively (Fig. 1A,B). Using an sPD-L1 threshold of 


\begin{tabular}{|c|c|c|c|c|}
\hline \multicolumn{2}{|c|}{ Characteristics in STS patients } & \multirow{3}{*}{$\begin{array}{l}\mathbf{N}(\mathbf{8 6}) \\
7 \\
39\end{array}$} & \multirow{3}{*}{$\begin{array}{l}\text { sPD-L1 average (SD) } \\
55.2(35.3) \\
69.4(77.3) \\
\end{array}$} & \multirow{3}{*}{$\begin{array}{l}\text {-valu } \\
0.698\end{array}$} \\
\hline \multirow{2}{*}{ Sex } & Male & & & \\
\hline & Female & & & \\
\hline \multirow{2}{*}{ Age } & $\leq 60 \mathrm{y}$ & 29 & $50.9(32.7)$ & \multirow{2}{*}{$* 0.342$} \\
\hline & $>60 y$ & 57 & $67.2(67.3)$ & \\
\hline \multirow{2}{*}{ Tumor size } & $\leq 10 \mathrm{~cm}$ & 42 & $58.6(40.8)$ & \multirow{2}{*}{$* 0.949$} \\
\hline & $>10 \mathrm{~cm}$ & 44 & $64.6(71.4)$ & \\
\hline \multirow[t]{2}{*}{ Location } & Extremity & 61 & $61.0(60.9)$ & \multirow{2}{*}{$* 0.977$} \\
\hline & Trunk & 25 & $63.3(52.1)$ & \\
\hline \multirow{2}{*}{ Tumor depth } & Superficial & 13 & $75.5(62.2)$ & \multirow{2}{*}{$* 0.539$} \\
\hline & Deep & 73 & $59.2(57.6)$ & \\
\hline \multirow{2}{*}{ Histological grade } & Low grade & 27 & $52.6(40.9)$ & \multirow{2}{*}{$* 0.098$} \\
\hline & High grade & 59 & $65.8(64.5)$ & \\
\hline \multirow{3}{*}{ Stage } & I & 27 & $52.6(40.9)$ & \multirow{3}{*}{${ }^{\#} 0.240$} \\
\hline & II & 15 & $59.8(43.6)$ & \\
\hline & III & 44 & $67.8(70.5)$ & \\
\hline \multirow{4}{*}{ Treatment } & Wide resection & 57 & $63.7(63.0)$ & \multirow{4}{*}{${ }^{\#} 0.527$} \\
\hline & Marginal resection & 24 & $55.9(43.3)$ & \\
\hline & Intralesional resection & 3 & $87.9(92.8)$ & \\
\hline & Ion beam radiotherapy: & 2 & $32.0(17.4)$ & \\
\hline \multirow{2}{*}{ Chemotherapy } & - & 60 & $64.5(65.7)$ & \multirow{2}{*}{0.951} \\
\hline & + & 26 & $54.9(35.6)$ & \\
\hline \multirow{2}{*}{ Radiotherapy } & - & 65 & $59.7(60.7)$ & \multirow{2}{*}{0.185} \\
\hline & + & 21 & $67.7(50.6)$ & \\
\hline \multirow{2}{*}{$\begin{array}{l}\text { History of other } \\
\text { malignant tumors }\end{array}$} & - & 68 & $59.2(40.6)$ & \multirow{2}{*}{0.316} \\
\hline & + & 18 & $70.8(101.8)$ & \\
\hline
\end{tabular}

Table 2. Characteristics of patients with STS. sPD-L1 values were compared for each parameter in STS patients. *Mann-Whitney test, ${ }^{\#}$ Kruskal-Wallis test.

\begin{tabular}{|l|l|l|l|l|}
\hline \multicolumn{2}{|l|}{ Characteristic } & n (86) & sPD-L1 average (SD) & p-value \\
\hline \multirow{2}{*}{ Recurrence } & - & 68 & $61.7(61.3)$ & \multirow{2}{*}{0.803} \\
\cline { 2 - 5 } & + & 18 & $61.4(46.4)$ & \\
\hline \multirow{2}{*}{ Metastasis } & - & 58 & $50.2(32.1)$ & \multirow{2}{*}{0.003} \\
\cline { 2 - 5 } & + & 28 & $85.3(87.3)$ & \multirow{2}{*}{0.016} \\
\hline \multirow{2}{*}{ Dead of disease } & - & 67 & $53.2(35.5)$ & \\
\cline { 2 - 5 } & + & 19 & $91.5(100.8)$ & \\
\hline
\end{tabular}

Table 3. sPD-L1 levels in cases of recurrence, metastasis, or dead of disease. In the period of this study, 19 patients had recurrence, 28 had metastases, and 19 were DOD. The sPD-L1 levels of patients with metastasis and DOD patients were significantly higher than those of patients without metastasis and patients who were not DOD, respectively, by the Mann-Whitney test.

$44.26 \mathrm{pg} / \mathrm{mL}$ based on Youden's index, the sensitivity and specificity for identifying metastasis were $85.7 \%$ and $56.9 \%$, respectively, and for DOD they were $84.2 \%$ and $50.7 \%$, respectively. Based on the ROC analysis, a cut-off value of $44.26 \mathrm{pg} / \mathrm{mL}$ was used to divide the groups into low $(\leq 44.26 \mathrm{pg} / \mathrm{mL})$ and high $(>44.26 \mathrm{pg} / \mathrm{mL}) \mathrm{sPD}-\mathrm{L} 1$ groups.

Logistic regression. To examine the associations of multiple factors for identifying recurrence, metastasis, or DOD, multiple logistic regression analyses were performed. No factors were significant for diagnosing recurrence. An sPD-L1 concentration greater than $44.26 \mathrm{ng} / \mathrm{mL}$ was associated with a significantly increased risk of metastasis and DOD (metastasis: OR 8.92, 95\%CI 2.63-30.0, P < 0.001; DOD: OR 5.84, 95\%CI 1.43-23.9, $\mathrm{P}=0.014$ ). Sex and tumor size, depth, and location were not related to the risk of metastasis or DOD (Table 4), whereas only age was related to DOD.

Prognostic analysis. Local recurrence-free survival (RS), metastasis-free survival (MS), and overall survival (OS) were compared between the low- and high sPD-L1 groups by Kaplan-Meier analysis and log-rank tests. RS showed no significant difference (5 years: low sPD-L1 79.6\%, high sPD-L1 65.1\%, P=0.205) (Fig. 2A). In only high-grade tumors, RS showed no significant difference $(5$ years: low sPD-L1 $=64.5 \%$, high sPD-L1 $=58.9 \%$, 

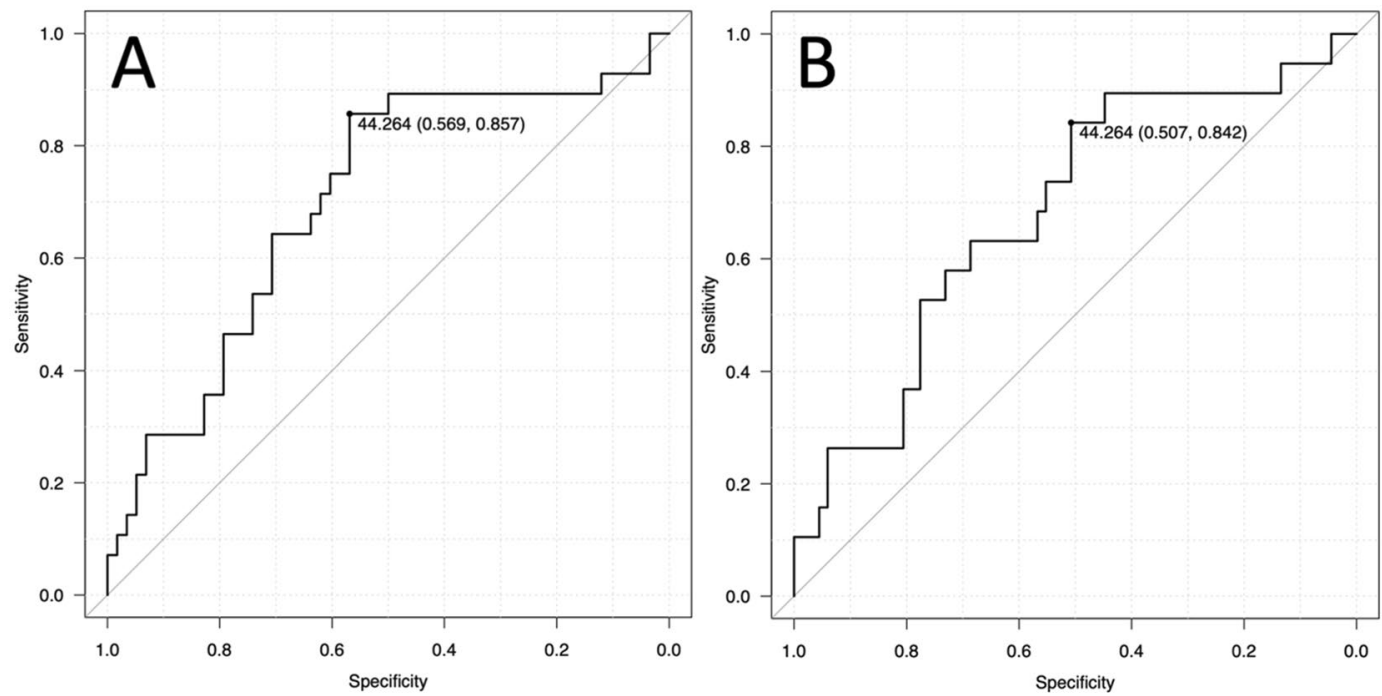

Figure 1. Receiver operating characteristic curve analysis. Diagnostic accuracy is evaluated by the area under the curve for identifying metastasis (A, AUC: 0.700, 95\% CI: 0.579-0.822) and DOD (B, right, AUC: 0.682, 95\% CI: $0.543-0.820)$. A cut-off of $44.26 \mathrm{pg} / \mathrm{mL}$ results in sensitivity of $85.7 \%$ and specificity of $56.9 \%$ for identifying metastasis and sensitivity of $84.2 \%$ and specificity of $50.7 \%$ for identifying DOD.

\begin{tabular}{|l|l|l|l|l|l|l|l|l|l|}
\hline \multirow{2}{*}{ Characteristic } & \multicolumn{4}{l}{ Recurrence } & \multicolumn{2}{l|}{ Metastasis } & \multicolumn{2}{l|}{ Dead of disease } \\
\cline { 2 - 11 } & OR & $\mathbf{9 5 \% C I}$ & p-value & OR & $\mathbf{9 5 \% C I}$ & p-value & OR & 95\%CI & p-value \\
\hline Male & 1.44 & $0.46-4.50$ & 0.528 & 0.53 & $0.19-1.49$ & 0.231 & 0.87 & $0.26-2.86$ & 0.828 \\
\hline Age & 1.02 & $0.98-1.07$ & 0.289 & 1.01 & $0.97-1.04$ & 0.688 & 1.07 & $1.01-1.13$ & $\mathbf{0 . 0 2 4}$ \\
\hline Size & 0.98 & $0.90-1.06$ & 0.645 & 0.95 & $0.89-1.03$ & 0.246 & 0.99 & $0.91-1.08$ & 0.906 \\
\hline Superficial & $2.28 \times 10^{-8}$ & $/$ & 0.992 & 0.70 & $0.16-3.07$ & 0.645 & 0.23 & $0.02-2.32$ & 0.215 \\
\hline Trunk & 2.80 & $0.87-8.91$ & 0.082 & 0.92 & $0.29-2.91$ & 0.896 & 2.29 & $0.65-7.97$ & 0.193 \\
\hline sPD-L1 >44.26 & 1.72 & $0.54-5.49$ & 0.355 & 8.92 & $2.63-30.0$ & $\mathbf{0 . 0 0 1}>$ & 5.84 & $1.43-23.9$ & $\mathbf{0 . 0 1 4}$ \\
\hline
\end{tabular}

Table 4. Multiple logistic regression analysis. Multiple logistic regression analysis to identify recurrence, metastasis, or dead of disease is shown. The ORs of sPD-L1 values were significant only in metastasis and DOD.

$\mathrm{P}=0.653$, Supplementary Fig. S4A). The high-sPD-L1 group had significantly lower MS (5 years: low sPD-L1 $88.4 \%$, high sPD-L1 42.4\%, P $<0.001$ ) (Fig. $2 \mathrm{~B}$ ). In only high-grade tumors, MS showed a significant difference (5 years: low sPD-L1 $=79.9 \%$, high sPD-L1 $=29.5 \%, \mathrm{P}=0.003$, Supplementary Fig. S4B). For OS, the high-sPD-L1 group had a significantly worse prognosis (5 years: low sPD-L1 $=89.2 \%$, high $\mathrm{sPD}-\mathrm{L} 1=64.1 \%, \mathrm{P}=0.011$ ) (Fig. 2C). In only high-grade tumors, OS showed a significant difference ( 5 years: low sPD-L1 $=81.4 \%$, high sPD-L1 $=5 \%, \mathrm{P}=0.040$, Supplementary Fig. S4C), as did MS.

Furthermore, to adjust for the imbalance in prognostic factors among patients, multivariate Cox proportional hazard analysis was used. For RS, only trunk location showed a significant difference on multivariate analysis. For MS, only the high-sPD-L1 group showed a significant difference (HR 5.66, 95\%CI 1.95-16.3, P=0.001). For OS, the high-sPD-L1 group, in addition to age, showed a significant difference on multivariate analyses (HR 5.04, 95\%CI 1.42-17.8, $\mathrm{P}=0.012$ ) (Table 5).

Immunohistology of PD-L1 for high-grade STS. PD-L1 immunohistological analysis was performed only for high-grade STSs. Of 59 high-grade STS cases, 6 cases were excluded due to tissue problems. Thus, a total of 53 patients were evaluated for histological positivity for cellular PD-L1 (more than $1 \%$ of membranous staining in tumor cells). Total positive staining for PD-L1 was observed in 16 patients $(30.1 \%)$. The positive staining rate in each histological subtype was $41.7 \%$ in UPS, $27.3 \%$ in MFS, $20 \%$ in DLS, $66.7 \%$ in MLS, $12.5 \%$ in LMS, $25 \%$ in SS, and 33.3\% in MPNST. The correlation between immunostaining and the SPD-L1 test was evaluated using the Kappa coefficient. All kappa values were below 0.351 , and there was no correlation between PD-L1 immunostaining and the sPD-L1 test (Table 6). Furthermore, there was no significant difference in sPD-L1 levels between groups staining positive and negative for PD-L1 (positive: 52.8 , negative $68.8, \mathrm{p}=0.437$, Mann-Whitney test, Supplementary Fig. S5A). Between the positive and negative immunostaining groups, RS (5 years: positive $52.5 \%$, negative $63.2 \%, \mathrm{P}=0.506$ ), MS (5 years: positive $42.2 \%$, negative $46.6 \%, \mathrm{P}=0.261$ ), and OS (5 years: positive $51.6 \%$, negative $55.0 \%, P=0.511$ ) were not significantly different on Kaplan-Meier analysis with the log-rank test (Supplementary Fig. S5B-D). 

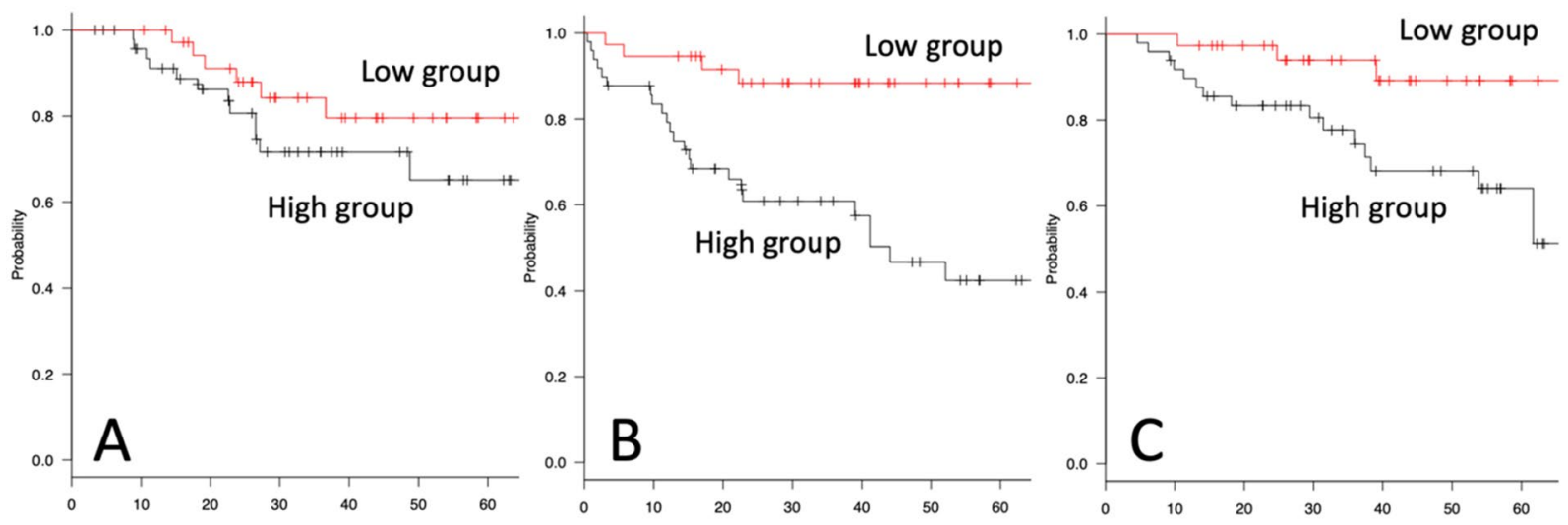

Figure 2. Kaplan-Meier analysis for STS. RS (A), MS (B), and OS (C) compared in the low- and high sPD-L1 groups are shown by Kaplan-Meier analysis. Five-year RS shows no significant difference (5 years: low sPD-L1 79.6\%, high sPD-L1 65.1\%, $\mathrm{P}=0.205$ ). The high-sPD-L1 group has significantly lower 5-year MS (low sPDL1 88.4\%, high sPD-L1 42.4\%, p >0.001). For 5-year OS, the high-sPD-L1 group has a significantly worse prognosis (low sPD-L1 $=89.2 \%$, high $\mathrm{SPD}-\mathrm{L} 1=64.1 \%, \mathrm{P}=0.011$ ). The $\mathrm{X}$-axis indicates months.

\begin{tabular}{|c|c|c|c|c|c|c|c|c|c|}
\hline & \multicolumn{3}{|l|}{ RS } & \multicolumn{3}{|l|}{ MS } & \multicolumn{3}{|l|}{ OS } \\
\hline & HR & $95 \% \mathrm{CI}$ & p-value & HR & $95 \% \mathrm{CI}$ & p-value & HR & $95 \% \mathrm{CI}$ & p-value \\
\hline Male & 1.24 & $0.47-3.26$ & 0.650 & 0.75 & $0.35-1.61$ & 0.470 & 0.90 & $0.36-2.26$ & 0.830 \\
\hline Age & 1.03 & $0.98-1.07$ & 0.150 & 1.01 & $0.98-1.04$ & 0.300 & 1.07 & $1.02-1.12$ & 0.004 \\
\hline Size & 0.97 & $0.91-1.05$ & 0.570 & 0.97 & $0.30-2.93$ & 0.350 & 1.01 & $0.94-1.07$ & 0.770 \\
\hline Super-ficial & $3.7110^{-9}$ & 1 & 0.990 & 0.94 & $0.30-2.93$ & 0.920 & 0.31 & $0.04-2.44$ & 0.260 \\
\hline Trunk & 2.68 & $1.02-6.99$ & 0.043 & 0.97 & $0.41-2.31$ & 0.950 & 1.48 & $0.56-3.87$ & 0.410 \\
\hline sPD-L1 > 44.2 & 1.98 & $0.72-5.42$ & 0.170 & 5.66 & $1.95-16.3$ & 0.002 & 5.04 & $1.42-17.8$ & 0.012 \\
\hline
\end{tabular}

Table 5. Multivariate COX proportional hazard analysis. For RS, only trunk lesions showed a significant HR. For MS, only high sPD-L1 showed a significant difference in the risk of metastasis. For OS, the HRs of sPD-L1 and age were significant.

\begin{tabular}{|c|c|c|c|c|c|}
\hline \multirow[b]{2}{*}{ High-grade STS } & \multirow[b]{2}{*}{ n (53) } & \multirow{2}{*}{$\begin{array}{l}\text { sPD-L1 group } \\
\text { High or low }\end{array}$} & \multicolumn{2}{|c|}{ PD-L1 immunostaining } & \multirow{2}{*}{$\begin{array}{l}\text { Kappa } \\
\text { coefficient }\end{array}$} \\
\hline & & & + & - & \\
\hline \multirow{2}{*}{ Total } & \multirow{2}{*}{53} & High & 9 & 22 & \multirow{2}{*}{-0.025} \\
\hline & & Low & 7 & 15 & \\
\hline \multirow{2}{*}{ UPS } & \multirow{2}{*}{12} & High & 4 & 3 & \multirow{2}{*}{0.351} \\
\hline & & Low & 1 & 4 & \\
\hline \multirow{2}{*}{ Myxofibrosarcoma } & \multirow{2}{*}{11} & High & 1 & 6 & \multirow{2}{*}{-0.294} \\
\hline & & Low & 2 & 2 & \\
\hline \multirow{2}{*}{ Liposarcoma (dedifferentiated) } & \multirow{2}{*}{10} & High & 0 & 5 & \multirow{2}{*}{-0.400} \\
\hline & & Low & 2 & 3 & \\
\hline \multirow{2}{*}{ Liposarcoma (myxoid) } & \multirow{2}{*}{3} & High & 1 & 0 & \multirow{2}{*}{0.400} \\
\hline & & Low & 1 & 1 & \\
\hline \multirow{2}{*}{ Leiomyosarcoma } & \multirow{2}{*}{8} & High & 1 & 4 & \multirow{2}{*}{0.157} \\
\hline & & Low & 0 & 3 & \\
\hline \multirow{2}{*}{ Synovial sarcoma } & \multirow{2}{*}{4} & High & 1 & 2 & \multirow{2}{*}{0.200} \\
\hline & & Low & 0 & 1 & \\
\hline \multirow{2}{*}{ MPNST } & \multirow{2}{*}{3} & High & 0 & 1 & \multirow{2}{*}{-0.500} \\
\hline & & Low & 1 & 1 & \\
\hline \multirow{2}{*}{ Others } & \multirow{2}{*}{2} & High & 1 & 1 & \multirow{2}{*}{0} \\
\hline & & Low & 0 & 0 & \\
\hline
\end{tabular}

Table 6. Comparison between immunostaining for PD-L1 and sPD-L1 levels in high-grade STSs. This table shows the comparison between positiv ity of immunostaining for PD-L1 and the level of high-sPD-L1 in highgrade STSs. Positivity of immunostaining for PD-L1 was defined as a positive stain rate of more than $1 \%$ of tumor cells. A high sPD-L1 was defined as a concentration greater than $44.26 \mathrm{pg} / \mathrm{mL}$. 


\section{Discussion}

The expression of PD-L1 in tumor tissues of various malignant tumors has been reported. PD-L1 expression was observed not only in tumor cells, but also in active T cells, B cells, NK cells, DCs, monocytes, macrophages, activated vascular endothelial cells, and mesenchymal stem cells ${ }^{22}$. To date, the upregulation mechanism of PD-L1 mRNA or PD-L1 protein was found to be via cytokines such as IFN- $\gamma$, IL-4, IL-6, IL-10, VEGF, and hypoxia-inducible factor-1 $\alpha$. The signal pathway of IFN- $\gamma / J A K 2 / I F N$, PI3K, and MEK/ERK/STAT1 can upregulate them ${ }^{23-25}$.

Since PD-L1 is a transmembrane protein, the relationships between PD-L1 expression on various cells in tumor tissues and prognosis have been reported in several malignant tumors. A few studies of PD-L1 in STS were mainly histopathological analyses. Positivity of PD-L1 was seen in $2.5 \%{ }^{26}, 11.7 \%^{27}, 12 \%{ }^{28}$, $43 \%$, and $64.8 \%^{29}$. In STS subtypes, positivity was seen as follows: UPS 14.9-82\%; myxoid liposarcoma 0-73\%; dedifferentiated liposarcoma 0-67\%; synovial sarcoma 0-75\%; leiomyosarcoma 0-70\%; MPNST 0-50\%; and myxofibrosarcoma $0-25 \%{ }^{27-30}$. In the present study, positive staining for PD-L1 was observed in 16 patients $(30.1 \%)$. PD-L1 positivity in STS subgroups varied widely. The present data for PD-L1 positivity were within the range previously reported. PD-L1 expression in tumor cells of STSs was not very intense. Some papers reported that PD-L1 positivity was a negative predictor of overall survival ${ }^{10,27,29}$, but other papers did not ${ }^{26,31,32}$. In the current study, there was no significant difference in prognosis between the PDL1-positive and PDL1-negative staining groups. Recently, in addition to cellular PD-L1, sPD-L1 has attracted attention, but there have been no reports about the prognosis in STS patients evaluated by serum SPD-L1 concentrations. The results of the present study successfully demonstrated the relationship between elevated sPD-L1 and a poor prognosis in STS. However, the sources of sPD-L1 were not elucidated.

Research into the source of sPD-L1 has progressed recently, and some important sources have been reported. First, one source is extracellular vesicles (EVs). Several studies reported PD-L1 on tumor-derived EVs including exosomes $^{33-37}$. Cancer cells can secrete a majority of their PD-L1 on exosomes, even with only slight cellular PD-L1 ${ }^{36,37}$. Basically, the amount of cellular PD-L1 and secretion of exosomal PD-L1 were different based on tumor cell types ${ }^{33-37}$. Interferon- $\gamma$ could increase secretion of PD-L1, including exosomes ${ }^{36}$. Exosomal PD-L1 secretion may be controlled by the tumor environment, including stimulation by cytokines. The second source is spliced variants. Zhou et al. showed spliced variants that lack the transmembrane domain in the culture medium, and they were observed in the plasma of melanoma patients ${ }^{38}$. The third source of sPD-L1 is proteolytic cleavage of membrane PD-L1. Chen et al. reported that the release of SPD-L1 into culture supernatant was decreased by a metalloproteinase inhibitor ${ }^{39}$. This means that matrix metalloproteinase (MMP) may release PD-L1 from the cell membrane. Although these are potent sources of sPD-L1, the possibility of other sources of sPD-L1, such as cell stress, cell injury, or cell death, cannot be excluded. In the present study, there was no correlation between the sPD-L1 test and PD-L1 immunostaining. Thus, it was unlikely that the source of sPD-L1 was cellular PD-L1 in STS cells. However, PC3 cells (prostate cancer cell line) and WM164 (melanoma cell line) had only slight cellular PD-L1, but secreted abundant exosomal PD-L1, and exosomal PD-L1 was increased by interferon- $\gamma^{36,37}$. Although cellular PD-L1 in STS cells was limited, STS cells could not be excluded as a source of elevated sPD-L1 by considering the additional effect of cytokines or the nature of STS cells.

Functional assessment of sPD-L1 is extremely important. Several studies supported the notion that exosomal PD-L1 inhibited IL-2 release and killing of tumor cells by T cells. Exosomal PD-L1 injection exacerbated transplanted tumor, and inhibiting the release of exosomal PD-L1 from tumor cells could decrease tumor growth ${ }^{33,36,37}$. Takeuchi et al. developed a unique ELISA to detect SPD-L1 that possessed binding capacity to PD-1 by using $\mathrm{PD}-1$-Ig fusion protein for capturing SPD-L1 ${ }^{40}$. This ELISA can differentiate SPD-L1 that binds membrane PD-1 from types that do not. Additionally, spliced variants of SPD-L1 show inhibitory functions on T-cell activation and proliferation ${ }^{38}$. Thus, the notion that circulating SPD-L1 has the potential to induce systemic immune suppression has been supported. In the present study, sPD-L1 had a strong relationship with metastasis and DOD in STS patients. Once the combination of high SPD-L1 and malignancy occurred, it led to worse MFS and OS in the high-sPD-L1 group than in the low-sPD-L1 group. An sPD-L1 $>44.26 \mathrm{pg} / \mathrm{mL}$ can predict future metastasis and a poor prognosis. High sPD-L1 was strongly related to metastasis and a poor prognosis. Thus, sPD-L1 may have potential to exacerbate tumor behavior in STS.

There have been some clinical trials of checkpoint inhibitors for sarcomas. In a small phase 2 study, six patients with synovial sarcoma were treated with ipilimumab. Time to progression ranged from 0.47 to 2.1 months (median 1.85 months), and overall survival was from 0.77 to 19.7 months (median 8.75 months) ${ }^{41}$. In another phase 2 study involving 80 patients with bone sarcomas or STSs treated with pembrolizumab, 7 (18\%) of 40 patients with STSs and $2(5 \%)$ of 40 patients with bone sarcomas showed objective responses ${ }^{32}$. In the most recent phase 2 study, 85 patients with bone sarcomas and STSs were treated with nivolumab with or without ipilimumab. The response rate was $5 \%$ in the nivolumab monotherapy group (43 patients) and $16 \%$ in the nivolumab and ipilimumab combination group (42 patients). Median overall survival was 10.7 months in the monotherapy group and 14.3 months in the combination group ${ }^{42}$. A clinical study of bone sarcoma and STS patients treated by checkpoint blockade therapy has just begun. These studies did not include evaluation of expressions of checkpoint molecules in the enrollment criteria. The authors pointed out the need to develop predictive biomarkers to establish which patients with sarcoma are most likely to benefit from checkpoint blockade, because, in the clinical data of treatment with checkpoint inhibitors, patients received benefits from this therapy regardless of PD-1 expres$\operatorname{sion}^{32,42-46}$. The present study showed that SPD-L1 concentrations could predict future metastasis and prognosis. Since sPD-L1 had a strong relationship with tumor aggravation, high sPD-L1 in STS patients may be a target for treatment by checkpoint inhibitors.

This retrospective study has some limitations. The number of patients was small, and subtypes could not be analyzed statistically because soft tissue tumors including sarcomas had over 20 histological subtypes, and the incidence rate of STS was low; thus, many studies must analyze STS as a whole entity, not by each histological 
classification. More blood samples within each histological subtype and longitudinal measurements may give us a more accurate assessment of the functional location of sPD-L1 in STS. We believe that the measurement of sPD-L1 may be useful for identifying metastases and poor outcomes in patients with STS.

\section{Materials and Methods}

Patients. A total of 135 patients with primary STSs who visited Mie University Hospital from 2009-2016 were enrolled in this study. Patients who had local recurrence or who were referred for additional resection after inadequate resection in a previous hospital or who had distant metastasis at the first visit were excluded from this study. Written, informed consent was obtained from each patient. For patients below the age 19 years, informed consent was obtained from their parents or legal guardian. This study was approved by the Ethics Committee of the Mie University Graduate School of Medicine. All procedures performed in studies involving human participants were in accordance with the ethical standards of the Ethics Committee of Mie University and with the Helsinki declaration of 1975. The histopathological diagnosis and histological grade were verified by independent pathologists. Clinical stage was classified according to the $7^{\text {th }}$ edition of the American Joint Committee on Cancer (AJCC) classification of STSs.

sPD-L1 measurement. Blood samples were obtained from all patients before biopsy or treatment. To remove remaining cells, serum tubes were centrifuged at $1500 \mathrm{~g}$ for $10 \mathrm{~min}$ at $4^{\circ} \mathrm{C}$. The serum samples were aliquoted and stored at $-80^{\circ} \mathrm{C}$.

Serum PD-L1 levels were measured quantitatively by enzyme immunoassay. On the measurement day, stored serum samples were thawed, and $100 \mu \mathrm{L}$ of serum were used for further analysis. Levels of PD-L1 were measured using a commercially available sandwich enzyme-linked immunosorbent assay (Human PD-L1 ELISA Kit, ab214565, Abcam, Cambridge, MA) according to the manufacturer's recommendations. The minimum detectable level of sPD-L1 was $2.91 \mathrm{pg} / \mathrm{mL}$; values under the detectable level were assigned a value of $0 \mathrm{pg} / \mathrm{mL}$.

PD-L1 immunohistological analysis for high-grade STS. After being deparaffinized in xylene and rehydrated in alcohol, to retrieve the antigenicity of PD-L1, hydrated heating in 1 mM EDTA buffer ( $\mathrm{pH} 8.0)$ was performed in a pressure cooker (Clipso $4 \mathrm{~L}$; T-FAL, Rumily, France) for $10 \mathrm{~min}$. After the sections were left at room temperature to cool in the soaking solution for $30 \mathrm{~min}$, the sections were incubated with anti-PD-L1 (E1L3N) XP rabbit monoclonal antibody (CST, Danvers, MA) at a dilution of 1:200. Antibody was diluted in $1 \%$ BSA/TBS to suppress the nonspecific reaction. After washing with tris-buffered saline (TBS), endogenous peroxidase was inactivated by $0.3 \%$ hydrogen peroxide in methanol for $30 \mathrm{~min}$. The sections were incubated with the reagent, peroxidase-labeled anti-rabbit immunoglobulin (DAKO, Glostrup, Denmark). The peroxidase was then intensified using fluorescyl-tyramide and anti-fluorescein conjugate HRP included in the CSA II Biotin-free Tyramide Signal Amplification System (DAKO). The reaction products were visualized in $0.15 \mathrm{mg} / \mathrm{mL}$ $3,3^{\prime}$-diaminobenzidine tetrahydrochloride (DAB) solution containing hydrogen peroxide. After washing in water, specimens were counterstained with hematoxylin. An individual pathologist evaluated PD-L1-positive cells. A tumor with membranous staining of more than $1 \%$ of tumor cells was considered positive for PD-L1 expression.

Statistical analysis. Statistical analysis was performed to compare the serum sPD-L1 levels to various clinical parameters using the Mann-Whitney $U$-test or the Kruskal-Wallis test for quantitative data. To evaluate the threshold for detecting recurrence, metastasis, or death due to disease, receiver operating characteristic (ROC) curve analysis was performed. The ROC curves were created by plotting sensitivity on the $y$-axis and the false-positive rate (1-specificity) on the $\mathrm{x}$-axis, and the area under the curve (AUC) was assessed. Local recurrence-free survival (RS) was defined as the time from the initial treatment to the date of clinically documented local recurrence. Metastasis-free survival (MS) was defined as the time from the initial treatment to the date of clinically documented distant metastasis. Overall survival (OS) was defined as the time from the initial treatment to the date of death attributed to the neoplasm. Kaplan-Meier survival plots and log-rank tests were used to assess the differences of RS, MS, and OS. The correlation between immunostaining and sPD-L1 test results was evaluated by the kappa coefficient test. To adjust for the imbalance in prognostic factors among patients, Cox proportional hazard analysis was used. $\mathrm{P}<0.05$ was considered significant. The EZR software program was used for statistical analyses ${ }^{47}$.

Received: 2 December 2019; Accepted: 11 May 2020;

Published online: 03 June 2020

\section{References}

1. Judson, I. et al. Doxorubicin alone versus intensified doxorubicin plus ifosfamide for first-line treatment of advanced or metastatic soft-tissue sarcoma: a randomised controlled phase 3 trial. The lancet oncology 15, 415-423 (2014).

2. Keir, M. E. et al. Tissue expression of PD-L1 mediates peripheral T cell tolerance. Journal of Experimental Medicine 203, 883-895 (2006).

3. Dong, H. et al. Tumor-associated B7-H1 promotes T-cell apoptosis: a potential mechanism of immune evasion. Nature medicine $\mathbf{8}$, 793 (2002).

4. Blank, C. \& Mackensen, A. Contribution of the PD-L1/PD-1 pathway to T-cell exhaustion: an update on implications for chronic infections and tumor evasion. Cancer immunology, immunotherapy 56, 739-745 (2007).

5. Wang, A. et al. The prognostic value of PD-L1 expression for non-small cell lung cancer patients: a meta-analysis. European journal of surgical oncology 41, 450-456 (2015).

6. Darb-Esfahani, S. et al. Prognostic impact of programmed cell death-1 (PD-1) and PD-ligand 1 (PD-L1) expression in cancer cells and tumor-infiltrating lymphocytes in ovarian high grade serous carcinoma. Oncotarget 7, 1486 (2016).

7. Thompson, R. H. et al. Tumor B7-H1 is associated with poor prognosis in renal cell carcinoma patients with long-term follow-up. Cancer research 66, 3381-3385 (2006).

8. Gadiot, J. et al. Overall survival and PD-L1 expression in metastasized malignant melanoma. Cancer 117, 2192-2201 (2011). 
9. Muenst, S. et al. Expression of programmed death ligand 1 (PD-L1) is associated with poor prognosis in human breast cancer. Breast cancer research and treatment 146, 15-24 (2014).

10. Kim, C. et al. Prognostic implications of PD-L1 expression in patients with soft tissue sarcoma. BMC cancer 16, 434, https://doi. org/10.1186/s12885-016-2451-6 (2016).

11. Frigola, X. et al. Identification of a soluble form of $\mathrm{B} 7-\mathrm{H} 1$ that retains immunosuppressive activity and is associated with aggressive renal cell carcinoma. Clinical Cancer Research 17, 1915-1923 (2011).

12. Finkelmeier, F. et al. High levels of the soluble programmed death-ligand (sPD-L1) identify hepatocellular carcinoma patients with a poor prognosis. Eur J Cancer 59, 152-159, https://doi.org/10.1016/j.ejca.2016.03.002 (2016).

13. Chang, B. et al. The correlation and prognostic value of serum levels of soluble programmed death protein 1 (sPD-1) and soluble programmed death-ligand 1 (sPD-L1) in patients with hepatocellular carcinoma. Cancer Immunol Immunother 68, 353-363, https:// doi.org/10.1007/s00262-018-2271-4 (2019).

14. Yoshida, J. et al. Clinical significance of soluble forms of immune checkpoint molecules in advanced esophageal cancer. Med Oncol 36, 60, https://doi.org/10.1007/s12032-019-1285-x (2019).

15. Okuma, Y. et al. High plasma levels of soluble programmed cell death ligand 1 are prognostic for reduced survival in advanced lung cancer. Lung cancer (Amsterdam, Netherlands) 104, 1-6, https://doi.org/10.1016/j.lungcan.2016.11.023 (2017).

16. Takahashi, N. et al. Serum levels of soluble programmed cell death ligand 1 as a prognostic factor on the first-line treatment of metastatic or recurrent gastric cancer. J Cancer Res Clin Oncol 142, 1727-1738, https://doi.org/10.1007/s00432-016-2184-6 (2016).

17. Shigemori, T. et al. Soluble PD-L1 Expression in Circulation as a Predictive Marker for Recurrence and Prognosis in Gastric Cancer: Direct Comparison of the Clinical Burden Between Tissue and Serum PD-L1 Expression. Ann Surg Oncol 26, 876-883, https://doi. org/10.1245/s10434-018-07112-x (2019).

18. Fan, Y. et al. Exosomal PD-L1 Retains Immunosuppressive Activity and is Associated with Gastric Cancer Prognosis. Ann Surg Oncol, https://doi.org/10.1245/s10434-019-07431-7 (2019).

19. Tominaga, T. et al. Clinical significance of soluble programmed cell death-1 and soluble programmed cell death-ligand 1 in patients with locally advanced rectal cancer treated with neoadjuvant chemoradiotherapy. Plos One 14, e0212978, https://doi.org/10.1371/ journal.pone.0212978 (2019).

20. Shen, H. et al. Soluble programmed death-ligand 1 are highly expressed in peripheral T-cell lymphoma: a biomarker for prognosis. Hematology 24, 392-398, https://doi.org/10.1080/16078454.2019.1590965 (2019).

21. Rossille, D. et al. High level of soluble programmed cell death ligand 1 in blood impacts overall survival in aggressive diffuse large B-Cell lymphoma: results from a French multicenter clinical trial. Leukemia 28, 2367-2375, https://doi.org/10.1038/leu.2014.137 (2014).

22. Zou, W. \& Chen, L. Inhibitory B7-family molecules in the tumour microenvironment. Nature Reviews Immunology 8, 467 (2008)

23. Dong, Y., Sun, Q. \& Zhang, X. PD-1 and its ligands are important immune checkpoints in cancer. Oncotarget 8, 2171 (2017).

24. Ota, K. et al. Induction of PD-L1 expression by the EML4-ALK oncoprotein and downstream signaling pathways in non-small cell lung cancer. Clinical cancer research 21, 4014-4021 (2015).

25. Wölfle, S. J. et al. PD-L1 expression on tolerogenic APCs is controlled by STAT-3. European journal of immunology 41, 413-424 (2011).

26. Torabi, A., Amaya, C. N., Wians, F. H. Jr. \& Bryan, B. A. PD-1 and PD-L1 expression in bone and soft tissue sarcomas. Pathology 49, 506-513, https://doi.org/10.1016/j.pathol.2017.05.003 (2017).

27. Que, Y. et al. PD-L1 Expression Is Associated with FOXP3+ Regulatory T-Cell Infiltration of Soft Tissue Sarcoma and Poor Patient Prognosis. J Cancer 8, 2018-2025, https://doi.org/10.7150/jca.18683 (2017).

28. D'Angelo, S. P. et al. Prevalence of tumor-infiltrating lymphocytes and PD-L1 expression in the soft tissue sarcoma microenvironment. Hum Pathol 46, 357-365, https://doi.org/10.1016/j.humpath.2014.11.001 (2015).

29. Kim, J. R. et al. Tumor infiltrating PD1-positive lymphocytes and the expression of PD-L1 predict poor prognosis of soft tissue sarcomas. PLoS One 8, e82870, https://doi.org/10.1371/journal.pone.0082870 (2013).

30. Pollack, S. M. et al. T-cell infiltration and clonality correlate with programmed cell death protein 1 and programmed death-ligand 1 expression in patients with soft tissue sarcomas. Cancer 123, 3291-3304, https://doi.org/10.1002/cncr.30726 (2017).

31. Zhu, Z. et al. Prognostic value of programmed death-ligand 1 in sarcoma: a meta-analysis. Oncotarget 8, 59570 (2017).

32. Tawbi, H. A. et al. Pembrolizumab in advanced soft-tissue sarcoma and bone sarcoma (SARC028): a multicentre, two-cohort, singlearm, open-label, phase 2 trial. The Lancet Oncology 18, 1493-1501 (2017)

33. Yang, Y. et al. Exosomal PD-L1 harbors active defense function to suppress $\mathrm{T}$ cell killing of breast cancer cells and promote tumor growth. Cell Res 28, 862-864, https://doi.org/10.1038/s41422-018-0060-4 (2018).

34. Theodoraki, M. N., Yerneni, S. S., Hoffmann, T. K., Gooding, W. E. \& Whiteside, T. L. Clinical Significance of PD-L1(+) Exosomes in Plasma of Head and Neck Cancer Patients. Clinical cancer research: an official journal of the American Association for Cancer Research 24, 896-905, https://doi.org/10.1158/1078-0432.Ccr-17-2664 (2018).

35. Ricklefs, F. L. et al. Immune evasion mediated by PD-L1 on glioblastoma-derived extracellular vesicles. Sci Adv 4, eaar2766, https:// doi.org/10.1126/sciadv.aar2766 (2018).

36. Chen, G. et al. Exosomal PD-L1 contributes to immunosuppression and is associated with anti-PD-1 response. Nature 560, 382-386, https://doi.org/10.1038/s41586-018-0392-8 (2018).

37. Poggio, M. et al. Suppression of Exosomal PD-L1 Induces Systemic Anti-tumor Immunity and Memory. Cell 177, 414-427.e413, https://doi.org/10.1016/j.cell.2019.02.016 (2019).

38. Zhou, J. et al. Soluble PD-L1 as a Biomarker in Malignant Melanoma Treated with Checkpoint Blockade. Cancer Immunol Res 5, 480-492, https://doi.org/10.1158/2326-6066.CIR-16-0329 (2017).

39. Chen, Y. et al. Development of a sandwich ELISA for evaluating soluble PD-L1 (CD274) in human sera of different ages as well as supernatants of PD-L1+ cell lines. Cytokine 56, 231-238 (2011).

40. Takeuchi, M. et al. Soluble PD-L1 with PD-1-binding capacity exists in the plasma of patients with non-small cell lung cancer. Immunol Lett, https://doi.org/10.1016/j.imlet.2018.01.007 (2018).

41. Maki, R. G. et al. A Pilot Study of Anti-CTLA4 Antibody Ipilimumab in Patients with Synovial Sarcoma. Sarcoma 2013, 168145, https://doi.org/10.1155/2013/168145 (2013).

42. D’Angelo, S. P. et al. Nivolumab with or without ipilimumab treatment for metastatic sarcoma (Alliance A091401): two open-label, non-comparative, randomised, phase 2 trials. The Lancet Oncology (2018).

43. Kaufman, H. L. et al. Avelumab in patients with chemotherapy-refractory metastatic Merkel cell carcinoma: a multicentre, singlegroup, open-label, phase 2 trial. The Lancet Oncology 17, 1374-1385 (2016).

44. Herbst, R. S. et al. Pembrolizumab versus docetaxel for previously treated, PD-L1-positive, advanced non-small-cell lung cancer (KEYNOTE-010): a randomised controlled trial. The Lancet 387, 1540-1550 (2016)

45. Weber, J. S. et al. Nivolumab versus chemotherapy in patients with advanced melanoma who progressed after anti-CTLA-4 treatment (CheckMate 037): a randomised, controlled, open-label, phase 3 trial. The Lancet Oncology 16, 375-384 (2015).

46. Rosenberg, J. E. et al. Atezolizumab in patients with locally advanced and metastatic urothelial carcinoma who have progressed following treatment with platinum-based chemotherapy: a single-arm, multicentre, phase 2 trial. The Lancet 387, 1909-1920 (2016).

47. Kanda, Y. Investigation of the freely available easy-to-use software 'EZR' for medical statistics. Bone Marrow Transplant 48, 452-458, https://doi.org/10.1038/bmt.2012.244 (2013). 


\section{Author contributions}

K.A. wrote the main manuscript text and prepared all figures and tables. K.A., T.N., Y.A., T.H., K.K. and K.N. explained this study to all patients and obtained their informed consent. T.O. performed sPD-L1 ELISA. T.I. performed immunostaining of PD-L1. A.H. evaluated the immunopathological analysis of PD-L1. A.S. supervised this study.

\section{Competing interests}

The authors declare no competing interests.

\section{Additional information}

Supplementary information is available for this paper at https://doi.org/10.1038/s41598-020-65895-0.

Correspondence and requests for materials should be addressed to K.A.

Reprints and permissions information is available at www.nature.com/reprints.

Publisher's note Springer Nature remains neutral with regard to jurisdictional claims in published maps and institutional affiliations.

(c) Open Access This article is licensed under a Creative Commons Attribution 4.0 International

License, which permits use, sharing, adaptation, distribution and reproduction in any medium or format, as long as you give appropriate credit to the original author(s) and the source, provide a link to the Creative Commons license, and indicate if changes were made. The images or other third party material in this article are included in the article's Creative Commons license, unless indicated otherwise in a credit line to the material. If material is not included in the article's Creative Commons license and your intended use is not permitted by statutory regulation or exceeds the permitted use, you will need to obtain permission directly from the copyright holder. To view a copy of this license, visit http://creativecommons.org/licenses/by/4.0/.

(c) The Author(s) 2020 